\title{
Erratum to: Incidence of periprocedural myocardial infarction and cardiac biomarker testing after percutaneous coronary intervention in Japan: results from a multicenter registry
}

Takahide Arai - Shinsuke Yuasa - Hiroaki Miyata - Akio Kawamura • Yuichiro Maekawa - Shiro Ishikawa - Shigetaka Noma - Soushin Inoue • Yuji Sato $\cdot$ Shun Kohsaka $\cdot$ Keiichi Fukuda

Published online: 26 May 2013

(C) Springer Japan 2013

Erratum to: Heart Vessels

DOI 10.1007/s00380-012-0314-x

Unfortunately, there was an error in the third sentence of "Procedures and data collection" under the section "Patients and methods" in the original article published. The correct sentence should be:

"We analyzed the data from 3894 patients undergoing PCI at 14 Japanese hospitals participating in the JCD-KICS (Japan Cardiovascular Database-Keio Interhospital Cardiovascular Studies) from September 2008 to August 2011.”

The online version of the original article can be found under doi:10.1007/s00380-012-0314-x.

T. Arai $(\bowtie) \cdot$ S. Yuasa · A. Kawamura · Y. Maekawa .

S. Kohsaka - K. Fukuda

Department of Cardiology, Keio University School of Medicine,

35 Shinanomachi, Shinjuku-ku, Tokyo 160-8582, Japan

e-mail: tarai@cpnet.med.keio.ac.jp

H. Miyata

Department of Healthcare Quality Assessment, Graduate School

of Medicine, University of Tokyo, Tokyo, Japan

S. Ishikawa

Department of Cardiology, Saitama City Hospital,

Saitama, Japan

\section{S. Noma}

Department of Cardiology, Saiseikai Utsunomiya Hospital,

Utsunomiya, Japan

S. Inoue

Department of Cardiology, Hino City Hospital, Tokyo, Japan

Y. Sato

Center for Clinical Research, Keio University School of

Medicine, Tokyo, Japan 